\title{
Aspect extraction performance with POS tag pattern of dependency relation in aspect- based sentiment analysis
}

\begin{abstract}
The most important task in aspect-based sentiment analysis (ABSA) is the aspect and sentiment word extraction. It is a challenge to identify and extract each aspect and it specific associated sentiment word correctly in the review sentence that consists of multiple aspects with various polarities expressed for multiple sentiments. By exploiting the dependency relation between words in a review, the multiple aspects and its corresponding sentiment can be identified. However, not all types of dependency relation patterns are able to extract candidate aspect and sentiment word pairs. In this paper, a preliminary study was performed on the performance of different type of dependency relation with different POS tag patterns in pre-extracting candidate aspect from customer review. The result contributes to the identification of the specific type dependency relation with it POS tag pattern that lead to high aspect extraction performance. The combination of these dependency relations offers a solution for single aspect single sentiment and multi aspect multi sentiment cases.
\end{abstract}

Keyword: Aspect extraction; Dependency relation; POS tag patterns; Extraction rule; Aspect-based sentiment analysis 